Chirurg 2021 · 92:1075-1076

https://doi.org/10.1007/s00104-021-01534-2

Angenommen: 12. Oktober 2021

(c) Springer Medizin Verlag $\mathrm{GmbH}$, ein Teil von Springer Nature 2021

Redaktion

C. Bruns, Köln

\section{Multimodale Therapie des Ösophaguskarzinoms}

\author{
Christiane J. Bruns
}

Klinik und Poliklinik für Allgemein-, Viszeral-, Tumor- und Transplantationschirurgie, Universitätsklinikum Köln, Köln, Deutschland
Die multimodale Behandlung des Ösophaguskarzinoms reicht von der endoskopischen Abtragung von Frühkarzinomen bis zur palliativen Systemtherapie im metastasierten Stadium oder definitiven Radiochemotherapie bei irresektablen Tumoren. In Deutschland werden jährlich ca. 7100 Ösophaguskarzinome erstdiagnostiziert, das macht ungefähr 1\% aller Malignome in Europa aus. Histologisch manifestiert sich das Ösophaguskarzinom in der Regel als Adeno- (AC) oder Plattenepithelkarzinom („esophageal squamous cell carcinoma“, ESCC) mit einer deutlichen Zunahme von Adeno- gegenüber den Plattenepithelkarzinomen. Die Therapiewahl wird - neben patientenspezifischen Faktoren - von Lage und Ausbreitung des Tumors, von Histologie und Metastasierungsstatus bestimmt.

Bei sog. Frühkarzinomen hängt das therapeutische Konzept (endoskopische Abtragung oder chirurgische Resektion) maßgeblich von der Infiltrationstiefe und damit vom Risiko einer lymphatischen Metastasierung ab. So ist die endoskopische Resektion das therapeutische Verfahren der Wahl unter kurativer Intention bei mukosalen Adenokarzinomen mit niedrigem Risiko (Grading 1 oder 2, keine Blut-/Lymphgefäßinvasion). Unter speziellen Voraussetzungen können auch Adenokarzinome der oberen Submukosa (sm1) endoskopisch abgetragen werden. Alle weiteren Stadien bedürfen einer chirurgischen Resektion. Beim Plattenepithelkarzinom ohne Risikofaktoren ist ab einer Infiltration der 3. Mukosaschicht (m3) bereits die chirurgisch-onkologische Ösophagusresektion indiziert. Bei guter Indikation weist die endoskopische Submukosadissektion (ESD) hohe Raten an
En-bloc- und R0-Resektionen selbst bei großen Läsionen (Schlosser T. et al.).

\section{॥) Multimodale Therapiekonzepte verbessern das Langzeitüberleben signifikant}

Bei ungefähr einem Drittel der Patienten wird die Erstdiagnose in einem resektablen Stadium gestellt, in dem ein kurativer Therapieansatz besteht. Mit alleiniger Resektion kann jedoch lediglich ein 5-Jahres-Überleben von ca. $20 \%$ erreicht werden. Multimodale Therapiekonzepte verbessern das Langzeitüberleben insbesondere beim lokal fortgeschrittenen, nichtmetastasierten Ösophaguskarzinom in den Stadien T3-T4 N-/+ M0 im Vergleich zur alleinigen chirurgischen Resektion signifikant. Hierbei stehen ergänzend zur kurativ intendierten chirurgischen Resektion die präoperative Radiochemotherapie oder bei Ösophagusadenokarzinomen und Adenokarzinomen des ösophagogastralen Übergangs (AEG) die perioperative Chemotherapie zur Verfügung. Die chirurgische Resektion sollte in den multimodalen Therapieschemata 4 bis 8 Wochen nach Ende der neoadjuvanten Therapie durchgeführt werden und beeinflusst dann die postoperative Morbidität und Mortalität im Vergleich zur alleinigen Operation nicht. Die postoperative Mortalität in den aktuellen multimodalen Protokollen beträgt 4-8\%. Patienten mit einem lokal fortgeschrittenen Ösophaguskarzinom nach neoadjuvanter Radiochemotherapie und Resektion, die eine residuelle Erkrankung nach R0-Resektion aufweisen, können zukünftig unabhängig vom histologischen Subtyp und 
der PD-L1 („,programmed cell death-ligand $1^{\prime \prime}$ )-Expression eine adjuvante Therapie mit Nivolumab über 12 Monate erhalten. Insgesamt korreliert das Ansprechen auf eine Immuntherapie mit der Tumormutationslast. Bekannte prognostischen Marker wie PD-L1, EBV (Epstein-Barr-Virus), MSI (Mikrosatelliteninstabilität) könnten somit in Zukunft durch die Bestimmung der Tumormutationslast verbessert und ergänzt werden (Lorenzen S. et al.).

Für das lokal fortgeschrittene Adenokarzinom des Ösophagus stehen mit der perioperativen Chemotherapie und der neoadjuvanten Radiochemotherapie zwei konkurrierende, jeweils evidenzbasierte und der alleinigen chirurgischen Behandlung überlegene Therapiekonzepte zu Verfügung. Eine signifikante Anzahl von Patienten zeigt nach der Therapie in den modernen neoadjuvanten Protokollen eine lokoregionäre Komplettremission des Tumors im Operationsresektat. Aktuell werden in europäischen prospektivrandomisierten „Non-inferiority“-Studien mit onkologischem Endpunkt die Möglichkeiten organerhaltender Konzepte bei klinischer Komplettremission („surgery as needed ", ",watch and wait") untersucht. Außerhalb von Studien haben diese Konzepte allerdings noch keine Rechtfertigung für eine Anwendung in der klinischen Routine. Wichtig ist auch, dass Salvageresektionen beim Tumorrezidiv nach definitiver Radiochemotherapie zwar mit guten onkologischen Ergebnissen durchgeführt werden können, allerdings mit deutlich erhöhter Morbidität einhergehen, sodass diese Eingriffe in Zentren durchgeführt werden sollten (Höppner J. et al.).

\section{॥ Die Immuntherapie hat in der palliativen Therapie einen wesentlichen Stellenwert}

Die Prognose des metastasierten Ösophaguskarzinoms ist nach wie vor sehr unbefriedigend. Auch hier wird die Immuntherapie einen wesentlichen Stellenwert in der palliativen Therapie des Ösophaguskarzinoms besitzen. Neben der kürzlich erfolgten FDA(U.S. Food and Drug Administration)-Zulassung von Pembrolizumab in Kombination mit Chemotherapie in der palliativen Erstlinientherapie für Adenound Plattenepithelkarzinome unabhängig von der PD-L1-Expression erfolgte in Europa die Zulassung der Immuntherapie in der palliativen Erstlinientherapie bei Tumoren mit einem PD-L1-CPS-Score von $\geq 10$ (Lorenzen S. et al.).

Im kurativen und meist multimodalen Setting ist die thorakoabdominelle Ösophagektomie mit Schlauchmagenhochzug das operative Standardverfahren. Dieser zunehmend minimal-invasiv sowie roboterunterstützt durchgeführte 2-Höhlen-Eingriff ist chirurgisch anspruchsvoll und führt durch Umsetzung von Mindestmengenregelungen in den kommenden Jahren zur Zentralisierung. Dieser Eingriff geht auch heute noch mit einer postoperativen Morbidität von bis zu $60 \%$ einher. Eine weitere entscheidende, in der multizentrischen internationalen CARDIA-Studie zu beantwortende Frage ist die optimale chirurgische Behandlungsstrategie der AEG-II-Tumoren (klassische Kardiakarzinome): transhiatal erweiterte Gastrektomie oder abdominothorakale Ivor-Lewis-Ösophagektomie.

Neben der Weiterentwicklung der roboterunterstützten minimal-invasiven Chirurgie stellt die Indocyaningrün(ICG)gestützte Fluoreszenzlaparoskopie mit intraoperativer Quantifizierung der Interponatsdurchblutung eine medizintechnische Innovation dar. Dies erscheint insbesondere zur Objektivierung oder Vorhersage möglicher Interponatsischämien nützlich (Schmidt T. et al.).

Korrespondenzadresse

Univ.-Prof. Dr. med. Christiane J. Bruns Klinik und Poliklinik für Allgemein-, Viszeral-, Tumor- und Transplantationschirurgie, Universitätsklinikum Köln

Kerpener Str. 62, 50937 Köln, Deutschland christiane.bruns@uk-koeln.de

Interessenkonflikt. C.J. Bruns gibt an, dass kein Interessenkonflikt besteht.

\section{Chirurgie Update 2022 Topaktuell und praxisnah}

4. und 5. März aus Mannheim 25. und 26. März aus Berlin

Umfangreiches Update-Wissen so flexibel wie noch nie: Das 14. DGAV-ChirurgieUpdate-Seminar findet als Hybridveranstaltung statt. Bilden Sie sich vor Ort mit Kolleginnen und Kollegen oder bequem von zuhause aus fort - die Zertifizierung ist für beide Formate beantragt.

Das Update-Konzept: Die wichtigsten Neuerungen des vergangenen Jahres aus der Allgemein- und Viszeralchirurgie werden unter der wissenschaftlichen Leitung von Prof. Dr. Dietmar Lorenz (Darmstadt), Prof. Dr. Helmut Friess (München) und Prof. Dr. Christoph-Thomas Germer (Würzburg) kritisch selektiert, analysiert und zusammengefasst. Die Relevanz für den Klinik- und Praxisalltag der Ärztinnen und Ärzte steht dabei im Vordergrund und der ausführlichen Diskussion über den Livechat mit den Referierenden wird viel Raum gegeben.

In diesem Jahr werden neben den Kerngebieten der Allgemein- und Viszeralchirurgie die Hot Topics »Gastrointestinale Onkologie«, »Perioperative Medizin Schmerztherapie«, »Kritische Fälle/Komplikationen« sowie »Bauchdeckenverschluss und Narbenhernien - Evidenz und Praxis (Video)« sowie das Special Topic: »Zertifizierung in der onkologischen Chirurgie:

Mehrwert oder nur Aufwand?« vorgestellt.

Zum Gesamtpaket der Teilnahme gehören neben den umfangreichen Seminarunterlagen der Download aller Vortragspräsentationen und die Vorträge im Nachgang als Video-on-Demand.

Weitere Informationen zu Programm, Referierenden und zur Anmeldung: www.chirurgie-update.com

Veranstalter: med update $\mathrm{GmbH}$ Hagenauer Straße 53 65203 Wiesbaden 